Philosophie ANTIQUE

\section{Philosophie antique}

Problèmes, Renaissances, Usages

12 | 2012

Autour de la perception

\title{
Livio ROSSETTI, Le dialogue socratique
}

\section{Agnese Gaile-Irbe}

\section{OpenEdition \\ Journals}

Édition électronique

URL : https://journals.openedition.org/philosant/957

DOI : 10.4000/philosant.957

ISSN : 2648-2789

Éditeur

Éditions Vrin

\section{Édition imprimée}

Date de publication : 1 novembre 2012

Pagination : 304-307

ISBN : 978-2-7574-0400-3

ISSN : $1634-4561$

\section{Référence électronique}

Agnese Gaile-Irbe, «Livio rossettı, Le dialogue socratique », Philosophie antique [En ligne], 12 | 2012, mis en ligne le 01 novembre 2018, consulté le 02 décembre 2022. URL : http://journals.openedition.org/ philosant/957 ; DOI : https://doi.org/10.4000/philosant.957

\section{(c) $\underset{\mathrm{EY}}{(\mathrm{NQ})} \mathrm{\Theta O}$}

Creative Commons - Attribution - Pas d'Utilisation Commerciale - Pas de Modification 4.0 International - CC BY-NC-ND 4.0

https://creativecommons.org/licenses/by-nc-nd/4.0/ 
est le principal interlocuteur de Socrate est le récit non pas de son asservissement, mais au contraire de son émancipation en vue de la carrière politique.

En terminant, il faut malheureusement souligner que cet ouvrage compte plusieurs dizaines de coquilles, au moins une erreur de mise en page (p. 27), des références imprécises aux ouvrages cités, et des références bibliographiques incomplètes. J'ai relevé trois passages où G. D. attribue à un personnage une position qui est en fait exprimée ou défendue par un autre. Ainsi, il faut lire « Antisthenes » à la place de «Charmides » page 127, et à la place de «Aeschines » page 181 ; page 262, il faut lire «Ischomachus » à la place de «Isocrates ». Enfin, l'index des noms (p. 275-276) et celui des termes (p. 277-280) sont certes utiles, mais je déplore néanmoins l'absence d'un index locorum qui permettrait au lecteur de contrôler si G. D. a tenu compte de tous les passages pertinents pour chacun des thèmes qui sont abordés dans les six études qui composent ce livre.

Louis-André DORION.

Livio RossetTi, Le dialogue socratique, Éditions Les Belles Lettres, Paris, 2011 (collection « Encre marine »), 292 p. ISBN 978-2-35088-041-9.

J'ai souvent l'impression que dans les domaines tels que les études platoniciennes où l'on ne fait que réfléchir sur les textes anciens qui ont déjà attiré un nombre immense de générations de lecteurs posant les mêmes questions, ce ne sont pas tant les idées ou les hypothèses qui déterminent la qualité d'une approche que la manière dont elles sont exprimées. La recherche du Socrate historique, la volonté de prouver qu'on peut le trouver dans les textes des socratiques si différents les uns des autres, ou la déconstruction des dialogues de Platon pour y dévoiler les stratagèmes manipulateurs, sont une entreprise extrêmement difficile. Pour convaincre le lecteur de ses intuitions et du bien-fondé de son approche, l'auteur doit construire tout un système rhétorique, exposer ses arguments d'une manière très nuancée et surtout trouver un langage qui puisse rendre compte d'un phénomène aussi étrange et multiforme que le dialogue socratique sans paraître déplacé, réducteur ou anachronique. Le livre de Rossetti accomplit admirablement cette tâche et dans son cas l'élégance et le bon goût manifestés dans la manière de s'exprimer deviennent aussi le bon goût et l'élégance de la pensée.

Ce recueil contient huit articles ou chapitres dont quatre ont déjà été publiés en français. Deux d'entre eux concernent Xénophon, deux autres - Platon et le reste - différents aspects de la littérature socratique, comme le ridicule ou le vocabulaire du philosophos. Pourtant, avant d'aborder ce choix d'articles, le lecteur doit savoir que Rossetti a produit une quantité impressionnante de publications dans le domaine concerné (son site web dénombre quelques 230 titres). Il n'est donc pas surprenant que, dans ce livre, l'auteur auquel il renvoie le plus souvent soit luimême. Il n'est pas surprenant non plus qu'à force de travailler sur les mêmes sujets et de reprendre ses travaux antérieurs (ses premières études sur les socratiques datent des années 1970), il ait créé en quelque sorte son propre monde, relativement clos. Pour le dire autrement, tout en étant conscient des positions divergentes d'autres historiens, il ne s'engage pas dans un débat avec eux, et tout porte à croire que Rossetti n'est pas quelqu'un qu'on pourrait amener facilement à changer d'avis. 
Le premier chapitre, qui forme une sorte d'introduction, présente une étude du phénomène même des logoi sokratikoi. Dans une présentation bien structurée, Rossetti montre l'importance quantitative d'un genre littéraire nouveau. Son analyse des sources conclut qu'environ quatorze disciples de la première génération des socratiques ont composé pas moins de deux cents ouvrages dont plus de la moitié ont présenté les traits de logoi sokratikoi. Ces textes ont été publiés pour la plupart dans les premières décennies du $\mathrm{IV}^{\mathrm{e}}$ siècle, ce qui donne un nouveau dialogue socratique par mois pendant un quart de siècle. Les listes qui nous sont parvenues des écrits des philosophes anciens nous ont habitués à la très grande productivité des anciens (comme le stoïcien Chrysippe qui a écrit plus de 700 livres à lui seul : D. L. VII, 180), mais Rossetti a raison de parler d'un « phénomène culturel de taille » et d'une production massive, car à cette époque-là Athènes n'avait connu que le flux régulier des pièces de théâtre.

L'exploration des témoignages transmis par les dialogues eux-mêmes lui permet de parler d'un processus de «standardisation » de ce logos avec des thèmes habituels et une manière codifiée de conduire l'entretien (avec, par exemple, la tradition de rapporter les entretiens entendus). Un chapitre corollaire précise une autre nouveauté que cette floraison des textes a dû apporter et permet de situer le dialogue socratique par rapport à l'activité des sophistes et des rhéteurs. Par une série d'exemples, Rossetti montre que les termes philosophos et philosophein ne sont devenus monnaie courante qu'au temps de la floraison des dialogues socratiques. Les sources ne permettent pas de distinguer un groupe d'intellectuels appelés philosophoi avant l'activité des socratiques et, ce qui est plus important, rien ne permet de dire que les sophistes comme Gorgias se soient qualifiés eux-mêmes de « philosophes »; et avec raison, dit Rossetti, car ils ont poursuivi d'autres objectifs.

Il faut donc se demander ce qu'était cet acte de philosophein inauguré par Socrate. L'analyse des stratégies communicationnelles du Socrate dépeint par plusieurs auteurs nous livre un personnage remarquablement cohérent. C'est cette conclusion-là qui permet à Rossetti d'entrer dans le grand débat de la « question socratique ». Il prend le contre-pied du mouvement sceptique qui à travers l'influence de Louis-André Dorion, exercée avant tout par son introduction magistrale au premier tome de l'édition CUF des Mémorables, semble prévaloir dans la communauté scientifique ${ }^{1}$. Selon Rossetti, Socrate se consacra avant tout à la construction de provocations intellectuelles, et c'est justement sur sa manière caractéristique de conduire l'entretien que les témoignages apportés par les dialogues sont concordants ${ }^{2}$. Par exemple, le chapitre III, 8 des Mémorables montre comment Aristippe a voulu appliquer l'elenchos à Socrate lui-même et cette stratégie est ouvertement qualifiée de socratique. Le Socrate historique serait donc à chercher du côté du comment et non pas du quoi. Si l'on peut se permettre une comparaison,

1. La position nihiliste quant à la possibilité de retrouver les traits du Socrate historique a été réaffirmée tout dernièrement dans un autre article écrit par L.-A. Dorion pour le Cambridge Companion to Socrates, édité par D. R. Morrison, 2011.

2. Pour les dialogues de Platon, il adopte la répartition proposée par G. Vlastos, avec une première période mettant en scène un Socrate plus ou moins authentique (p. 30 et 219). 
cet approche du problème socratique m'a rappelé l'hypothèse de Michael Gagarin selon laquelle l'élément commun à tous les systèmes légaux des poleis grecques, introuvable si l'on compare les lois elles-mêmes, serait à chercher du côté de la procédure ${ }^{3}$.

Le chapitre VI décrit ce que Rossetti appelle « le projet macro-rhétorique » de Socrate, c'est-à-dire les procédés qu'il emploie pour enfermer l'interlocuteur dans une situation rassurante qu'il ne peut pas contrôler avant de l'acculer dans une impasse (p. 227-231). Pour décrire cette macro-rhétorique, Rossetti emploie plusieurs notions empruntées à l'informatique, comme le «formatage » et la « saturation » (p. 233-235). La comparaison entre la rhétorique de Socrate et celle de Gorgias permet de mieux cerner les différences : contrairement à Gorgias, Socrate ne dévoile jamais son objectif; il cherche non seulement à réfuter son partenaire mais à bouleverser sa personnalité tout entière ; il veut provoquer des émotions - le choc, l'embarras et la honte, etc. Le cas le plus représentatif de cette technique sans pitié est probablement le chapitre IV, 2 des Mémorables où Socrate encercle Euthydème. Rossetti est persuadé que les socratiques n'ont rien inventé de nouveau mais ont développé un exercice souvent pratiqué en compagnie de Socrate.

Dans les pages consacrées à l'étude de Xénophon, Rossetti adopte une attitude qui me paraît très sensée. Sans suivre le mouvement inauguré par Leo Strauss et présenter Xénophon comme un penseur d'une profondeur inouïe, Rossetti parle de « deux Xénophon »- l'un qui est vif et pénétrant et l'autre qui ne profère que des généralités; il est regrettable que le second fasse souvent obstacle à l'appréciation du premier (p. 118). La qualité de la dramaturgie dans le chapitre IV, 2 des Mémorables est telle que Rossetti a raison d'appeler ce morceau « l'Euthydème de Xénophon », comme s'il s'agissait d'une contrepartie au dialogue de Platon.

Dans les chapitres consacrés à Platon, Rossetti approfondit l'examen des « règles du jeu» des entretiens socratiques et souligne l'importance de l'atmosphère qui enveloppe ces textes. Cette atmosphère est un facteur tellement important de l'assentiment du lecteur que la fausseté de certains arguments échappe, ditil, même aux commentateurs. Rossetti produit une liste des caractéristiques qui passent souvent inaperçues, comme certaines phrases de transition trop rapides, la réticence des interlocuteurs prise pour acquiescement, les formules qui demandent l'autorisation de ne pas développer davantage un argument, la clause « dans la mesure du possible » et le déséquilibre intellectuel entre Socrate et ses interlocuteurs. Il nous alerte, somme toute, sur «la nécessité de se méfier de Platon » (p. 259). Pour arriver à la doctrine il serait nécessaire de dépouiller les dialogues de tout ce qu'il y a « autour », mais cette tâche s'avère très ardue. Il serait néanmoins inexact de parler à ce propos d'une découverte ou d'une lecture jamais considérée : voyez déjà certaines des règles d'interprétation de Platon proposées par Diogène Laërce (III, 65) qui vont dans le même sens.

L'article sur l'Euthyphron de Platon est de loin le plus long du livre et se présente comme une lente navigation à travers ce que Rossetti appelle les secrets de

3. M. Gagarin, « The Unity of Greek Law », dans D. Cohen, M. Gagarin (éd.), The Cambridge Companion to Ancient Greek Law, 2005, p. 29-40. 
Platon. Un des points de départ de l'interrogation est un court passage surprenant au début de la conversation - c'est la manière très injuste dont Socrate résume le premier embryon de définition de la piété proposé par Euthyphron (5d8-e3); celui-ci avait essayé de cerner une catégorie des actes pieux, mais Socrate réagit (6d1-3) comme s'il n'avait indiqué qu'un cas singulier ( «la piété, c'est ce que moi je fais »). L'examen des causes possibles de cette manipulation attribuée à Socrate engage Rossetti dans une discussion sur les dialogues aporétiques en général, sur le contexte et la leçon de l'entretien, sur la recherche définitionnelle qu'il considère comme un apport de Platon, ainsi que sur la datation et le cadre narratif. Il m'est impossible de résumer ici ses acquis sans faire tort à la manière très nuancée dont il les présente.

C'est un livre à la fois solide et courageux, écrit par un homme très intelligent.

Agnese GAILE-IRBE.

Suzanne Husson, La République de Diogène. Une cité en quête de la nature, Paris, Librairie philosophique J. Vrin, 2011 (Histoire des doctrines de l'Antiquité classique, 40), 235 p. ISBN 978-2-7116-2265-8.

On compte plusieurs études sur la Politeia attribuée à Diogène, mais, sauf erreur de ma part, aucun livre ne lui avait jusqu'à maintenant été consacré, non plus qu'à la pensée politique de Diogène. L'ouvrage passionnant que Suzanne Husson (désormais S. H.) vient d'y consacrer démontre à l'évidence que la pensée politique de Diogène, et des cyniques en général, méritait une étude approfondie. Dans le premier chapitre de l'ouvrage («Problèmes textuels », p. 21-45), S. H. se penche sur les principaux témoignages relatifs à la Politeia de Diogène, notamment le fameux témoignage de Philodème (Sur les stö̈ciens, P.Herc. 155 et $339=$ SSR V B 126), dont S. H. reproduit le texte (p. 22-23) et fournit une traduction (p. 2325). Après un examen détaillé et rigoureux des arguments pro et contra, elle conclut de façon convaincante à l'authenticité de la Politeia attribuée à Diogène ( $c f$. p. 40) et elle procède ensuite à un examen des parallèles entre la République de Platon et celle de Diogène. Ces parallèles sont suffisamment nombreux et étroits pour justifier la conclusion suivant laquelle la Politeia de Diogène dépend de la République de Platon et y répond en radicalisant les positions de ce dernier ( $c f$. p. 45 ; voir aussi p. 105-132: «Radicaliser Platon »).

L'un des grands mérites de l'ouvrage de S. H., et c'est surtout cet apport que je discuterai, est d'avoir pleinement reconnu l'importance de la recherche de l'autarcie dans le cynisme et d'avoir cherché à comprendre l'articulation entre la recherche de l'autarcie individuelle et le projet collectif d'une cité cynique ( $c f$., entre autres, «La cité de l'autarcie », p. 75-101). Il s'agit d'une question redoutable dont S. H. reconnaît d'entrée de jeu la difficulté ( $c f$. p. 7) : si le sage est autarcique et que la nature lui fournit d'elle-même tout ce dont il a besoin pour réaliser son autarcie, en quoi les cyniques ont-ils besoin d'une cité ? De plus, « n'est-il pas contradictoire dans les termes de vouloir constituer une cité composée d'individus autarciques? »(p.83). Il s'agit d'un vrai problème et un rapide rappel des positions de Platon et d'Aristote permettra de mesurer l'ampleur de la difficulté à laquelle est confrontée S. H. Dans la République, la nécessité pour l'homme de vivre en cité vient de ce que l'homme n'est pas autarcique sur le plan individuel et 